\title{
Habitus und Feld

\begin{abstract}
Anregungen für eine Neuorientierung der geographischen Entwicklungsforschung auf der Grundlage von Bourdieus «Theorie der Praxis»
\end{abstract}

\section{Thomas Dörfler, Olivier Graefe, Detlef Müller-Mahn, Bayreuth}

\section{Zur Kritik an «Entwicklung» und «Handeln»}

Die entwicklungstheoretische Diskussion leidet seit Anfang der neunziger Jahre unter Verunsicherung und Mutlosigkeit (Elsenhans 1996, 1997). Zur Verunsicherung tragen der Disput um das «Scheitern der großen Theorie» bei (Menzel 1992), aber auch die Enttäuschung anfänglich zu optimistischer Erwartungen gegenüber der Globalisierung und daran anschließend die beunruhigende Erkenntnis, dass nachholende Entwicklung für einen großen Teil der Menschheit gegenwärtig aussichtsloser erscheint denn je (KREUTZMANN 2002; Scholz 2002). Die Mutlosigkeit der Theoriediskussion äußert sich darin, dass ausgerechnet unter dem Einfluss der Globalisierung ein Rückzug in «Theorien mittlerer Reichweite» angetreten wird, verbunden mit einer Renaissance entwicklungspragmatischer Konzepte und einer Abkehr von den vermeintlich gescheiterten Großtheorien (HeIN 1997).

In der geographischen Entwicklungsforschung fiel diese Trendwende der Theoriediskussion auf fruchtbaren Boden, weil sie die Forschungsperspektive auf überschaubare Kontexte reduzierte und den Forschungsgegenstand näher an die räumliche Mikroebene heranbrachte, den Bereich also, der für empirische Fallstudien am ehesten handhabbar ist. Beispiele hierfür sind die Ansätze zur Neuen Institutionenökonomie und zur livelihood-Forschung. Zudem wurde bei der Suche nach neuen theoretischen Orientierungen die in der Sozialgeographie bereits seit Ende der achtziger Jahre diskutierte Handlungstheorie GidDENsscher Prägung (WerLen 1995 und 2000) für die geographische Entwicklungsforschung entdeckt. Mit ihrem methodologischen Individualismus bzw. «methodologischen Subjektivismus» (WERLEN 1999: 258) bot sie eine praktikable Grundlage für Untersuchungen, die das Handeln ausgewählter Akteure in den Mittelpunkt stellen (z.B. Neuburger 2002; Tröger 2002; Müller-Mahn 2001).

Bei kritischer Betrachtung dieser neueren Ansätze und ihrer empirischen Anwendung bleiben jedoch wesentliche Fragen offen, die über die im Kontext von Fallstudien erfassbare Akteursebene hinausweisen (BLOTeVogel 1999; Meusburger 1999): Wie autonom sind die Subjekte in ihrem Handeln? Inwiefern lässt sich aus dem beobachteten Handeln einzelner Individuen auf Prozesse und Probleme der Gesellschaft schließen? In welchem Zusammenhang stehen der untersuchte Fall und das Ganze? Ausschließlich akteurszentrierte Ansätze führen ebenso wie die Theorien mittlerer Reichweite zu einer Begrenzung der Perspektive und klammern damit einen wesentlichen Teil der Wirklichkeit aus. Unserer Meinung nach ist jedoch nicht eine Verengung der Perspektive gefragt, sondern eine Erweiterung, wenn es darum gehen soll, die soziale Realität von Menschen in Entwicklungsländern zu verstehen.

Diese methodischen Probleme sollen nachfolgend zunächst hinsichtlich der Paradigmen der Entwicklung und des Handelns kurz erläutert und am Beispiel des livelihood-Ansatzes illustriert werden. Im anschließenden zweiten Teil des Beitrags wird der gesellschaftstheoretische Entwurf des französischen Soziologen Pierre Bourdieu vorgestellt, speziell das Konzept von Habitus und Feld, um dann im dritten Teil zu diskutieren, inwieweit sich hier für die (geographische) Entwicklungsforschung Anregungen finden lassen, mit denen die eingangs skizzierten Begrenztheiten der normativ-präskriptiven Entwicklungsdebatte und der Theorien der mittleren Reichweite überwunden werden können. Anliegen dieses Artikels ist es nicht, über die Sinnhaftigkeit größerer oder kleinerer Entwicklungstheorien zu diskutieren, sondern vielmehr der Frage nachzugehen, wie die Forschung in Entwicklungsländern auf eine allgemeine Gesellschaftstheorie bezogen werden kann.

Entwicklung: ein neokoloniales Konzept? Entwicklung ist schon immer ein umstrittener Begriff gewesen, ein Begriff, der diffuse Wertungen transportiert, der konträre Positionen hervorbringt, aber Widersprüche unter ein und demselben Etikett verdeckt (SEERS 1974; Nohlen \& Nuscheler 1992; KöSSLER 1998). Die inhärenten Widersprüche sind bekannt, aber die kritische Auseinandersetzung mit den eigenen Prämissen wird in der Theoriediskussion unterschiedlich weit getrieben (SACHS 1989). Besonders radikal fällt die Kritik der post development studies aus, die sich nicht nur gegen den Begriff Entwicklung wendet, sondern insbesondere auch gegen die dahinter stehenden Weltbilder (vgl. zusammenfassend ZiaI 2001). Grundlage dafür bilden eine Reihe von nachfolgend aufgeführten Argumenten, die zwar auch früher schon diskutiert wurden, die aber mit neuen Konsequenzen verknüpft werden können: 
Erstens muss auf die analytische Unschärfe des Entwicklungsbegriffes hingewiesen werden, die aus seiner Bedeutungsvielfalt resultiert. Je nach Kontext und Standpunkt enthält Entwicklung implizit die Konnotation normativer Inhalte (Entwicklung als Verbesserung), den Verweis auf Prozesshaftigkeit und Dynamik (Entwicklung als Veränderung bzw. Wandel), auf den Nachvollzug einer vorgegebenen Richtung (Entwicklung als Fortschritt) oder auf die Gestaltung und Trägerschaft von Prozessen (Entwicklung als geplantes, zielorientiertes Programm). Es trägt nicht gerade zur Klarheit der Diskussion bei, wenn Entwicklung in Verbindung mit Adjektiven wie beispielsweise partizipativ oder armutsorientiert als positiv aufgeladener Begriff verwendet wird, in Verbindung mit den Begriffen fragmentierend oder krisenhaft aber etwas Negatives bezeichnet. Andererseits vermag der Begriff der Entwicklung sinnhafte Bezüge zwischen verschiedenen Prozessen herzustellen, er kann

«dem Ausdruck bloßer Veränderung eine Richtung [...] geben, die, ausgesprochen oder nicht, die Annahme eines Ziels einschließt» (Asendorf, Flemming et al. 1994: 165).

Damit ist er teleologisch-transzendent und hat tendenziell den Zweck, jegliche Veränderung als einen versöhnlichen Prozess immerwährender Verbesserung aufzufassen. Mit ihm ist die

«vielfach unterschwellige, aber keineswegs zwingende Auffassung verbunden, dass es sich hierbei um einen zwar unumkehrbaren, aber langsamen und allmählichen Vorgang über große Zeiträume hinweg handelt, so dass Entwicklung [...] als 〈wohltätiges〉 Gegenprinzip zur Revolution erscheint» (ibid).

Der zweite Kritikpunkt am Entwicklungsparadigma richtet sich gegen die Auffassung von der sog. Dritten Welt als räumlich-politischem Konstrukt, das der Heterogenität der länderspezifischen Situationen nicht gerecht wird (NoHLEN \& NusCHELER 1992; BOECKH 1992). Außerdem folgen Entwicklungstheorien, die sich auf den Unterschied zwischen sog. Erster und Dritter Welt beziehen, nach Auffassung der entwicklungskritischen Autoren unvermeidlich einem inhärenten Eurozentrismus bzw. normativen Modernismus (MвEmbe 2001: 7; Ziar 2001). Der Entwicklungsbegriff ist aufgeladen mit Residuen, die zum einen europäisch-okzidentaler Natur sind, geprägt durch jenes abstrakte Verlangen unserer Gesellschaftskonstellation nach «letzer» Wahrheit, die Foucault (2002) als «Wille zum Wissen» bezeichnet hat. Zum anderen sind sie ethisch-kultureller Art: jener Hang zur Berufung, sich als Heilsbringer für die «unzivilisierten» bzw. «unterentwickelten» Länder und Kontinente dieser Erde zu fühlen. Die im negativen Sinne folgenreichste Verquickung dieser beiden Traditionen brach sich im Kolonialismus Bahn.

Drittens schließlich wird kritisiert, dass Entwicklungstheorien, die die Dialektik von «entwickelt» und «unterentwickelt», von sog. Erster und Dritter Welt thematisieren, zumindest implizit eine hierarchisierende Sichtweise übernehmen, die «Unterentwicklung» in all ihren Erscheinungsformen im Verhältnis zu der Gegenseite, dem Zustand des «Entwickeltseins», definiert. Unterentwicklung ist ein Manko, egal, ob man sie als Rückständigkeit oder als Abhängigkeit konzeptionalisiert. Damit tragen Entwicklungstheorien zur Perpetuierung von hierarchischen Herrschaftsverhältnissen bei, selbst wenn sie deren Überwindung propagieren, weil sie stets die Hierarchie mitdenken.

«[...] Entwicklung - auch nachhaltige Entwicklung ist nur ein weiteres Schlüssel- und Schlagwort. Dieses Wort ist insofern fragwürdig, als es den ehemaligen Kolonialmächten die Fortsetzung ihrer «zivilisatorischen〉 Mission ermöglicht, diesmal aber mit komplizenhafter Unterstützung der lokalen Eliten, die nun ihrerseits die eigenen Völker hinters Licht führen und unterjochen. Der konzeptuelle Rahmen dieses Betrugs ist die liberal(istisch)e Globalisierung» (Traoré 2002: 15).

Entwicklung dient in dieser Sicht somit als neokoloniales Dispositiv, um den Anderen in sozialer und ökonomischer Abhängigkeit zu halten und in irgendeiner Form als rückständig zu klassifizieren (vgl. MвEMBE 2001): Eine Gesellschaft, die bei ihrer Entwicklung Hilfe braucht, läuft Gefahr, von den Gebern nicht nur als hilfsbedürftig betrachtet zu werden, sondern auch mit dem Makel der Unvollkommenheit oder Unfähigkeit belegt zu werden (MBEMBe 2001; Illich 1970). Es ist demnach paradoxerweise gerade der normative Aspekt des Entwicklungsbegriffes, der dem Theoriediskurs eine sublime «Logik der Verachtung» verleiht.

Die Folgerung aus diesen Kritikpunkten ist eine radikale Distanzierung vom Begriff der Entwicklung, dem manche Autoren gar jeglichen analytischen Gehalt absprechen (SACHS 1989), und von allen Entwicklungstheorien, denen die Unfähigkeit zum Denken jenseits von Abhängigkeiten vorgeworfen wird. Auch wenn man der radikalen Konsequenz der post development studies nicht in jedem Punkt folgt, ist doch der Hinweis auf die methodischen Defizite des entwicklungstheoretischen Diskurses nicht von der Hand zu weisen. Die Kritik deckt sich ein Stück weit mit dem eingangs konstatierten Unbehagen der Entwicklungstheorie gegenüber ihrem Gegenstand, der Entwicklung. Aus Sicht der geographischen Entwicklungsforschung stellt sich daran anschließend jedoch die Frage: Wenn nicht Entwicklung - was dann?

Handeln statt Entwicklung? Das Handeln individueller Akteure findet schon länger das Interesse der geographischen Forschung in Entwicklungsländern, als Beispiele seien die (Überlebens-)Strategien von Menschen in (verwundbaren) Gruppen (z.B. BoHLE 2001), das Handeln von Nomaden und gesellschaftlichen Aussenseitern (z.B. ScHOLz 1995) oder die Anpassung 
von Landnutzung und Lebensweise an natürliche, wirtschaftliche, soziale und politische Bedingungen genannt (z.B. KRINGs 1991). Erst seit Mitte der neunziger Jahre aber fanden die theoretischen Impulse von Giddens (1997) und WerLen (1995) auf dem Weg über die Sozialgeographie mit einiger Verzögerung auch Eingang in die geographische Entwicklungsforschung. $\mathrm{Ob}$ die Handlungstheorie hier auch einmal als «Königsweg» - wie in anderen Teilbereichen der Geographie - bezeichnet werden wird, bleibt abzuwarten (WIRTH 1998). Die Handlungstheorie GidDENsscher Prägung entspricht mit ihrem Fokus auf das handelnde Subjekt und auf die (Eigen-)Verantwortung des Einzelnen gegenüber der Gesellschaft durchaus dem Zeitgeist der neoliberalen Wende, aber eine unkritische Übertragung dieser Betrachtungsweise auf den Kontext von Gesellschaften in Entwicklungsländern erscheint höchst problematisch.

Die augenblickliche Konjunktur akteurszentrierter Ansätze bei der Untersuchung von Entwicklungsproblemen ist durchaus symptomatisch für die Verlagerung der Perspektive von gesellschaftlichen Zusammenhängen hin zu einer Fokussierung auf das eigenverantwortlich agierende Subjekt, von der Struktur zum Handeln. Handeln wird in dieser Perspektive als zwar kollektiv eingebunden, in letzter Konsequenz aber als individualisierte Kategorie verstanden (vgl. KRINGS \& MÜLLER 2001). In ihrer Ausformulierung entwicklungstheoretischer Prämissen und praxisnaher Modelle kommen diese Ansätze folglich zu einer Perspektivenverschiebung bei der Bewertung gesellschaftlicher Prozesse. Probleme der Entwicklung werden dabei gewissermaßen als Folge von Fehlhandlungen gesehen, d.h. von unangepassten, irrationalen oder ausgebliebenen Handlungen. Konsequenz dieser Verlagerung der Perspektive auf die mehr oder weniger individuelle Handlungsebene ist, dass die Verantwortung für Veränderung nicht mehr primär mit gesellschaftlichen und/oder makroökonomischen Strukturen verknüpft wird, sondern mit fähigen Akteuren vor Ort. Individuelle Akteure tragen gemäß dieser Sichtweise nicht nur die Verantwortung für ihre Entwicklung, sondern sie tragen auch die Risiken des Scheiterns. Die fehlende kritische Auseinandersetzung mit dieser Tendenz hat zur Folge, dass die dadurch konstituierten neuen Abhängigkeitsverhältnisse aus dem Blick geraten und damit latent perpetuiert werden.

Dadurch bleibt die von der gesellschaftlichen Realität ausgehende Gewalt, die Bedeutung der Struktur für das Handeln und die sich daraus ergebenden Handlungszwänge der Menschen in Entwicklungsländern unberücksichtigt. Die «systematische Unterbelichtung der Bedeutung von Institutionen und Organisationen» in WERLENS Entwurf zur handlungszentrierten Sozialgeographie wurde bereits an anderer
Stelle kritisch diskutiert (Blotevogel 1999: 22). Diese Kritik gilt mindestens ebenso für den Versuch, Entwicklungsprobleme mit dem Handeln autonomer Subjekte erklären zu wollen.

Die Vorstellung von frei bzw. rational gemäß ihren Ausstattungen handelnden Akteuren wie etwa in den livelihood-Ansätzen ist eine Fiktion: Rationalität im Handeln ist ausschließlich im Kontext der Bedingungen zu rekonstruieren, welche Subjekte mitsamt deren (rationalen) Motiven erst hervorbringen. Als analytische Folge ergibt sich hier eine epistemologische Umkehrung: die gesellschaftliche Formation wird nicht durch die mehr oder minder individuellen Handlungen erklärt, sondern die gesellschaftliche Formation erklärt die individuellen Handlungen mitsamt deren Motivationen, ohne dass sie dabei deterministisch wirken könnte. Dementsprechend steht nicht ein Individuum einer Struktur gegenüber, dessen Handeln einen rationalen und intendierten Akt darstellt (der freilich auch unbeabsichtigte Folgen haben kann), sondern Intention und Handlungsgründe müssen von der Objektseite her (also den Strukturen) abgeleitet werden.

Livelihood und Vulnerabilität - ein Beispiel aktueller Entwicklungsforschung. Ein Beispiel für neuere entwicklungspragmatische Ansätze mit handlungsorientierten Bezügen ist der livelihood-Ansatz. Er ist in engem Kontakt zur Entwicklungspraxis entstanden und bietet vor diesem Erfahrungshintergrund eine übersichtliche Anleitung für die empirische Forschung zu Fragen der Lebensabsicherung unter Bedingungen erhöhter Vulnerabilität. Seine Forschungsperspektive richtet sich auf existenzielle Notlagen und speziell auf Ressourcen und Strategien zu deren Bewältigung. Die Untersuchung alltäglicher Praktiken im Zeichen der Verwundbarkeit sozialer Gefüge und ökologischer Systeme versucht hier insbesondere an die (teilweise bereits eingespielten bzw. auf vorhandenes Wissen aufbauenden) Handlungsweisen der Subjekte anzuknüpfen und deren inhärente, quasi kulturellen Potentiale der Krisenbewältigung aufzuzeigen.

Die nicht zu bestreitende Leistungsfähigkeit der livelihood-Theoreme für eine konkrete empirische Forschungstätigkeit in kleinräumig-lokalen Kontexten und für Aufgaben der Projektplanung stößt aber an Grenzen, wenn es um weiterreichende Zusammenhänge geht: Der Ansatz liefert zwar ein Gerüst zur Beschreibung von existenzbedrohenden Situationen, ohne aber wirklich eine Erklärung für deren Zustandekommen bieten zu können. Das dem Ansatz zugrunde liegende Verständnis von Überlebenssituationen ist - aus nachvollziehbaren und für die Entwicklungspraxis durchaus auch sinnvollen Gründen - aktualitätsbezogen, dabei aber tendenziell ahistorisch und statisch. Seine Fragestellungen richten sich darauf, inwiefern die 
Überlebenssicherung gefährdet ist und wie Menschen darauf reagieren. Von der Position einer theoriegeleiteten geographischen Entwicklungsforschung aus sollte dagegen nicht allein das Inwiefern wichtig sein, sondern auch das Warum, d.h. beispielsweise die Ursachen von Vulnerabilität, oder die Dynamik von Abhängigkeitsverhältnissen sozialer Gefüge unter verwundbaren Bedingungen.

Der pragmatisch-empirische Umgang des Ansatzes mit Überlebenssituationen ist realitätsnah. Wenn mangels übergeordneter theoretischer Bezüge der soziale Raum, innerhalb dessen Überlebenssicherung stattfindet, nur auf Beschränkungen und Befähigungsaspekte hin untersucht wird, lassen sich wesentliche Beweggründe des Handelns nicht erfassen. Vielmehr verhindert geradezu die genannte Konzentration auf das autonome Handeln von Akteuren und Gruppen die Erforschung von Abhängigkeiten. Gemeint sind hier speziell ökonomische Zwänge (z.B. Eigentumsverhältnisse etc.), politische Machtverhältnisse und soziale Beziehungen. Widmet sich der livelihood-Ansatz diesen Bedingungen, so geschieht dies meist rein deskriptiv als Aufaddierung der mehr oder weniger vorhandenen Arten von Kapital bzw. des Zugangs dazu, was Handlungspotentiale vorbestimmt.

Zusammenfassung der Kritik. Um richtig verstanden zu werden: Der forschungspraktische Gebrauchswert von Theorien mittlerer Reichweite und ihr Nutzen für die Projektplanung werden an dieser Stelle nicht diskutiert. Unsere kritischen Anmerkungen weisen vielmehr darauf hin, dass sie die gesellschaftliche Dimension der in Entwicklungsländern ablaufenden Prozesse nicht adäquat erfassen können, dass sie auf einem verkürzten Handlungsverständnis beruhen und auch den Begriff der Entwicklung oftmals in naiver Weise verwenden. Sie können daher keinen Ersatz für «große» Theorie bieten, und vor allem nicht für eine Theorie der Gesellschaft. Schwachpunkte dieser neueren entwicklungstheoretischen Ansätze ergeben sich zum Einen aus dem normativen Erbe des Entwicklungsbegriffes selbst, und zum Anderen aus dem unzureichend fundierten Gesellschaftsverständnis und der undifferenzierten Übertragung handlungstheoretischer Ansätze in der Tradition von GidDENs auf die Situation in Entwicklungsländern.

An dieser Stelle soll deshalb ein anderer theoretischer Ansatz vorgestellt werden, von dem wir meinen, dass er eine Alternative bietet, um den Zusammenhang von individuellem Handeln und gesellschaftlichen Veränderungen in Entwicklungsländern zu verstehen, und dabei ohne den impliziten Verweis auf eine zu erfolgende Entwicklung und den dabei mitschwingenden Eurozentrismus auszukommen. Dadurch soll jener erwähnte «defizitäre» Blick vermieden werden, der im Anderen - letztendlich nur ein Teil von einem selber - immer einen Problemfall sieht, wie wir das dem Entwicklungsparadigma oben unterstellten. Wir plädieren für einen Perspektivenwechsel von der Entwicklungsforschung zur Sozialforschung in Entwicklungsländern. Dies glauben wir auf theoretischer Ebene mit der Soziologie Pierre Bourdieus begründen zu können.

\section{Die Logik der Praxis bei Bourdieu}

\subsection{Entstehungszusammenhang des Werkes}

Zum Verständnis der handlungstheoretischen Arbeiten des französischen Soziologen PIERre Bourdieu ist es hilfreich, zunächst einmal deren Entstehungszusammenhang zu betrachten. In seinen frühen Studien in und über Algerien in den 1960er Jahren (vgl. BourDIEU 2000) war Bourdieu noch eindeutig der Denkweise des Strukturalismus verhaftet. Bald aber wurde ihm bewusst, dass er sich damit in zu engen methodologischen Bahnen bewegte: Das kabylische Haus, dessen materielle Struktur er in einem später in selbstkritischer Intention noch einmal abgedruckten Aufsatz (Bourdieu 1979: 48-65) detailliert beschreibt, war ihm nur zugänglich, weil Frankreich damals in Algerien Krieg führte, und er konnte die Innenausstattung des Hauses nur deshalb so genau aufnehmen, weil das Dach abgebrannt war (BouRdieu 1987: 11). Vor dem historischen Hintergrund des Unabhängigkeitskrieges gelangte BourdiEU zu der Überzeugung, dass es nötig sei, nicht bei den oberflächlichen Strukturen stehenzubleiben, sondern sich intensiver mit den Bedingungen der wissenschaftlichen Erkenntnis auseinanderzusetzen und das «Verhältnis zum Objekt weiter zu objektivieren» (Bourdieu 1987: 7). Mit diesem Anliegen suchte er die in der öffentlichen Diskussion behauptete «Irrationalität» der algerischen Bevölkerung in einer schwierigen Transformationsphase besser zu verstehen, als es die etablierten ökonomischen und entwicklungstheoretischen Ansätze jener Zeit vermochten:

«Die Diskrepanz zwischen den in einer vorkapitalistischen Ökonomie geformten Handlungen und dem importierten [...] ökonomischen Kosmos führte unabweislich zu Bewusstsein, dass der Zugang zu den elementaren ökonomischen Verhaltensweisen sich keineswegs von selbst versteht und dass der <rational> genannte ökonomische Akteur das Produkt ganz besonderer historischer Umstände ist» (Bourdieu 2000: 7).

Durch seine Forschungen in Nordafrika kam BourDIEU zu der Erkenntnis, dass die Menschen in Entwicklungsländern in ihren Handlungsweisen keineswegs «rückständig» sind, sondern einen durchaus kreativen Umgang mit den sich rasch wandelnden sozioökonomischen Rahmenbedingungen zeigen. Der Rationalität des westlichen Forschers bzw. der seines 
Gesellschaftssystems steht nach Auffassung von BouRDIEU nicht das Irrationale bzw. Prämoderne der Menschen des Maghreb gegenüber, sondern es kommt vielmehr darauf an, die Rationalität und die inhärenten Handlungslogiken sogenannter traditioneller Gesellschaften unter veränderten Rahmenbedingungen herauszuarbeiten. Es geht ihm darum

«[...] die Praxis in ihren unscheinbarsten Formen zu erklären, den rituellen Handlungen, der Heiratswahl, dem ökonomischen Alltagsverhalten usw., und dabei sowohl den Objektivismus zu vermeiden, bei dem das Handeln als eine mechanische Reaktion ohne einen [Handelnden] verstanden wird, als auch den Subjektivismus, der das Handeln als die planvolle Ausführung einer bewussten Absicht bestimmt, als freien Entwurf eines Bewusstseins, das seine eigenen Zwecke setzt und seinen Nutzen durch rationales Kalkül maximiert» (BouRdiEU \& WACQUANT 1996:153).

Mit anderen Worten: Die Logik der Praxis erschließt sich nicht, wenn allein die Objektseite oder die Subjektseite betrachtet werden. Nur wenn die Beziehung zwischen beiden einbezogen wird, lässt sich beobachtetes Handeln sinnadäquat erklären. BouRdieus wissenschaftliches Programm, seine Theorie der Praxis, richtet sich auf eine Überwindung der Spaltung zwischen Objektivismus und Subjektivismus. Dabei kommt dem Konzept des Habitus eine besondere Bedeutung zu.

\subsection{Habitus und Feld}

Im folgenden Kapitel sollen einige Grundgedanken aus BouRDIEUs Handlungstheorie auf fünf Thesen verkürzt dargestellt werden, um den Versuch zu erläutern, Handeln im Verhältnis von Individuum und Gesellschaft zu begreifen und damit die gesellschaftstheoretischen Defizite des methodologischen Individualismus zu vermeiden (vgl. Tab. 1 und Abb. 1).

(1) Handeln wird nicht allein von einem bewussten Kalkül bestimmt, sondern auch von der Relation zwischen Subjekt und Gesellschaft.

Der erste Kernpunkt der Bourdieuschen Soziologie bezieht sich auf die Relation zwischen individuellen und kollektiven Subjekten. Diese Relation ist für die weitere Argumentation deshalb so wichtig, weil BourDIEU davon ausgeht, dass ein wesentlicher Teil des individuellen Handelns nicht bewusst-intentional erfolgt, trotzdem aber durchaus einer übergeordneten Richtung folgt, die sich nur über das spezifische Verhältnis zwischen Individuum und Gesellschaft erklären lässt. Die Relation Individuum-Gesellschaft geht über die unmittelbaren Interaktionen zwischen Subjekten hinaus, indem sie die dauerhaften Strukturen von sozialen Lagen und sozialen Positionen einbezieht, von Gegebenheiten also, die für Bourdieu «mehr Realität haben als die Subjekte, die sie verbinden» (Bourdieu et al. 1991: 21). Die Relation zwi- schen Subjekt und Gesellschaft ist objektiv insofern, als sie unabhängig vom Bewusstsein und Willen der Subjekte existiert (Bourdieu \& WACQUANT 1996: 127).

(2) Die Relation zwischen Subjekt und Gesellschaft ist von objektiven Machtbeziehungen und unreflektierten, verinnerlichten Dispositionen geprägt.

Das Verhältnis zwischen Subjekt und Gesellschaft wird auf gesellschaftlicher Ebene von ungleichen Kräfteverhältnissen und auf der Ebene der Subjekte von verinnerlichten Handlungsmustern und Orientierungen geprägt.

«In einer bestimmten sozialen Formation treten die Instanzen, die objektiv auf die legitime Ausübung einer symbolischen Durchsetzungsgewalt Anspruch erheben und aufgrund dessen das Monopol der Legitimität anstreben, notwendig in Konkurrenzbeziehungen, d.h. in Kräfteverhältnisse und symbolische Verhältnisse, deren Struktur ihrer Logik gemäß den Zustand des Kräfteverhältnisses zwischen den Gruppen oder den Klassen zum Ausdruck bringt» (Bourdieu et al. 1973: 29).

Hier wird also explizit die Perspektive über das Subjekt und sein Handeln hinaus auf gesellschaftliche Dominanz- und Abhängigkeitsverhältnisse ausgeweitet, die besonders auch für die entwicklungstheoretische Debatte wichtig sind.

Unter Dispositionen kann man die verinnerlichten Prägungen verstehen, auf eine bestimmte Weise wahrzunehmen, zu fühlen, zu denken und zu handeln. Sie sind dem Individuum aufgrund seiner objektiven Existenzbedingungen und sozialen Laufbahn gleichsam in Fleisch und Blut übergegangen, auch wenn sie ihm nur selten bewusst sind, so dass sie in konkreten Handlungssituationen unreflektiert befolgt werden können. Dispositionen sind erworbene/erlernte Wahrnehmungs-, Denk- und Handlungsweisen der Subjekte/ Akteure im Rahmen ihrer praktischen Erfahrungen in sozialen Feldern. Sie stellen durch die Erfahrung von und die Auseinandersetzung mit der Praxis der Sozialwelt herausgebildete Präferierungsmodi dar, die nicht intentional sind. Sie werden sowohl auf leiblicher wie auch auf kognitiver Ebene wirksam, indem sie dem Subjekt eine Art «begriffsloses Erkennen» ermöglichen und einen praktischen Sinn ausbilden.

(3) Die objektiven Machtbeziehungen konstituieren soziale Felder, innerhalb derer die Subjekte konkrete Positionen einnehmen, die sie nur durch den Einsatz des ihnen verfügbaren Kapitals verändern können.

Soziale Felder entstehen als Kontexte durch die Beziehungen der Subjekte, und zwar speziell durch die Konkurrenz um Ressourcen, Positionen oder Macht (Bourdieu \& WaCQuant 1996: 127). Sie sind cha- 


\section{Feld}

Mehrdimensionale Matrix des Sozialen: «Spielfeld», das durch spezifische Relationen zwischen den Subjekten, durch ihre Positionen und durch ihren «Einsatz» strukturiert wird.

\section{Dispositionen}

Präferierungsmodi, die sich durch die Erfahrungen und Auseinandersetzungen in der Praxis bestimmter sozialer Felder herausgebildet haben und ein «begriffsloses Erkennen» / einen «praktischen Sinn» ermöglichen.

\section{Habitus}

System von Dispositionen der Subjekte, die in Beziehung zu einem sozialen Feld erworben wurden. Der Habitus äussert sich in den unbewusst aufeinander abgestimmten, inkorporierten Orientierungen und Handlungskompetenzen der Individuen, d.h. in einer «Konzertierung ohne Dirigent».

Tab. 1: Die zentralen Begriffe bei Bourdieu

Central notions of BouRdiEU

Les concepts centraux, selon BOURDIEU

rakterisiert durch asymmetrische Machtverhältnisse, d.h. durch eine Hierarchie zwischen Dominanten und Dominierten. Das soziale Feld ist somit eine Art Matrix des Sozialen, konstituiert aus Räumen gesellschaftlich existenter Praktiken, d.h. der Auseinandersetzung um soziale Positionen, welche gekennzeichnet sind durch Strukturen und Relationen. Ein Feld stellt einen ausdifferenzierten sozialen Raum dar, in welchem Praktiken aktualisiert und erzeugt werden. Es ist ein gesellschaftlich wirksames Handlungsmuster, das nun Interessen und Strategien als handlungsleitende und -generierende subjektive Kategorien hervorbringt.

BouRdieu verwendet für den Begriff des Feldes auch die Metapher des Spiels (jeu):

«In der Tat lässt sich das Feld mit einem Spiel vergleichen [...]. So gibt es Einsätze bei diesem Spiel, [...] die im wesentlichen das Produkt der Konkurrenz der Spieler untereinander sind; eine Investition in das Spiel, eine Besetzung (im psychologischen Sinn) des Spiels [...]: Die Spieler sind im Spiel befangen, sie spielen, wie brutal auch immer, nur deshalb gegeneinander, weil sie alle den Glauben [...] an das Spiel und den entsprechenden Einsatz, die nicht weiter $\mathrm{zu}$ hinterfragende Anerkennung teilen [...], und dieses heimliche Einverständnis ist der Ursprung ihrer Konkurrenz und ihrer Konflikte» (BourdiEU \& WACQUANT 1996: 127ff.).

Das französische enjeu ist im Deutschen mit «Einsatz» nur ungenau übersetzt, denn gemeint ist hier wie im englischen «what's at stake» alles, «was auf dem Spiel steht».

Soziale Felder lassen sich nicht immer klar voneinan- der abgrenzen, da sie sich partiell überlagern können, und ein Subjekt an mehreren Spielen parallel teilnehmen kann. In seinen eigenen empirischen Untersuchungen befasste sich BouRdiEu exemplarisch mit den Feldern der Religion, der Literatur, der Wissenschaft, der Politik und der Ökonomie (KraIs \& Gebauer 2002: 34).

Im Zusammenhang mit dem Feld und seiner Strukturierung ist ein zweiter Gedanke wichtig, den BouRDIEU in die Theoriediskussion einbrachte, nämlich den des Kapitals und der verschiedenen Kapitalsorten. Darunter versteht er zunächst all das, was als Investition in das Spiel bzw. Feld eingebracht werden kann, was zur Markierung der eigenen Position innerhalb des Feldes dient und als Gratifikation für die Teilnehmer am Spiel bereitgehalten wird. Dabei sind verschiedene Sorten von Kapital zu unterscheiden, die miteinander ausgetauscht oder substituiert werden können, nämlich ökonomisches, soziales, kulturelles und symbolisches Kapital. Bei seinen Untersuchungen über die kabylische Gesellschaft zeigt BourdiEU, wie hier ökonomisches Kapital durch die Praktiken des Güteraustauschs in «sozialen Kredit» bzw. symbolisches Kapital verwandelt wird, und umgekehrt (Bourdieu 1979: 352 und 1987: 218). Das Feld der sozialen Beziehungen wird damit durch die Versuche zur Aneignung und Maximierung verschiedener Sorten von Kapital konstituiert. Wesentlich ist dabei, dass sowohl die aktuelle Verfügung wie auch der weitere Zugang zu bestimmten Kapitalsorten von der Position des Subjektes innerhalb der Machthierarchie des jeweiligen Feldes abhängen. Die Kapitalsorten präfigurieren Herkunft und Distinktionsvermögen der Gesellschaftsmitglieder, 


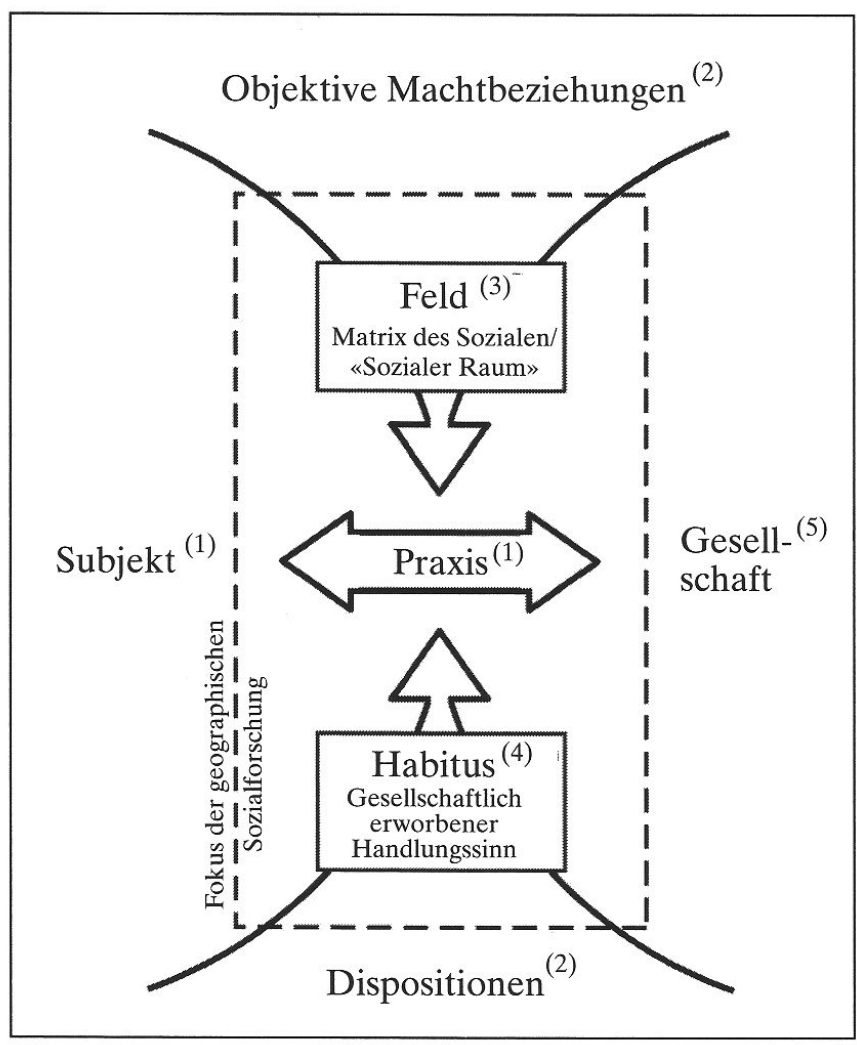

Für (1) - (5) siehe die Thesen im Text in Kap. 2.2.

Abb. 1: Begriff-schematische Darstellung der «Logik der Praxis»

Definition diagram of the «logic of practice»

Présentation conceptuelo-schématique de la «logique de la pratique»

Entwurf und Bearbeitung: D. MÜLlER-MahN, J. BREUNIG

ohne sie schlicht zu determinieren: vielmehr ist die Differenzierung einer Gesellschaft eine Folge des unterschiedlich erfolgreichen Strebens nach Kapitalaneignung.

(4) Der Habitus als System dauerhafter und übertragbarer Dispositionen führt zur unbewussten Anpassung von Vorstellungen, Praktiken und Strategien der Subjekte an eine übergeordnete Logik.

Das zentrale Konzept in der Handlungstheorie von Bourdieu ist das des Habitus. Gemeint ist damit ein «System von in Beziehung zu einem Feld erworbener Dispositionen» der Subjekte (Bourdieu 1992: 115), das zu einer unbewussten Abstimmung von Praktiken führt, bzw., in seinen eigenen Worten, einer «Konzertierung ohne Dirigent» (Bourdieu 1987: 110). Ein gemeinsamer Habitus ist die Voraussetzung dafür, dass soziales Handeln verschiedener Subjekte aufeinander abgestimmt werden kann, indem es derselben «Verhaltensgrammatik» folgt (KraIs \& GebaUer 2002: 32). Die das Handeln unbewusst leitenden Dispositionen zeichnen sich dadurch aus, dass sie von einer größeren Zahl von Menschen geteilt werden, und dass sie über längere Zeiträume erhalten bleiben. Sie bilden die «Erzeugungs- und Ordnungsgrundlagen für Praktiken und Vorstellungen, die objektiv an ihr Ziel angepasst sein können, ohne jedoch bewusstes Anstreben von Zwecken [...] vorauszusetzen» (BourdiEu 1987: 98).

Daraus ergibt sich das Paradox, dass Handlungen rational sein können, ohne dass das Subjekt notwendigerweise wissen müsste, warum dies so ist (im Gegenteil: diese Reflexivität wäre für weite Teile der Praxis geradezu hinderlich und handlungsvereitelnd).

In Bourdieus Begriff des Habitus sind die sozialen Strukturen unserer Subjektivität repräsentiert, welche sich durch unsere ersten Erfahrungen sowie in unserer weiteren Sozialisation bilden. Der Habitus drückt die Art und Weise aus, wie sich soziale Strukturen in unseren Köpfen durch die «Verinnerlichung der Äußerlichkeit» («Interiorisierung der Exteriorität») verfestigen (Bourdieu 1987: 102). Das Konzept ist jedoch nicht zu verwechseln mit Schicksal oder Tradition. Der Habitus umfasst die generalisierten Handlungskompetenzen von Individuen und regelt deren konformes Handeln innerhalb von sozialen Feldern. Diese Handlungskompetenzen sind klassenspezifisch ungleich verteilt, weil der Habitus ein «Produkt der gesamten individuellen Geschichte, [...] der gesamten kollektiven Geschichte der Familie und der Klasse» (ibid: 116) ist. Wichtig ist hier das Verhältnis von Habitus und Feld: Der Habitus bildet sich nur in einem Feld aus und disponiert so die sich daraus ableitenden Kompetenzen, die das Denken, Handeln und Wahrnehmen der Subjekte prägen. Das Verhältnis von Habitus und Feld ist durch eine spezifische Dialektik wechselseitiger Bestimmung gekennzeichnet: «Es liegt in der Relation zwischen Habitus und Feld, dass der Habitus dazu beiträgt, zu bestimmen, was ihn bestimmt» (BoHN 1991: 26). Während der Habitus also die «Verinnerlichung der Äußerlichkeit» darstellt, ist das Feld folglich die «Veräußerlichung der Innerlichkeit».

Um den Habitus als verinnerlichten oder praktischen Sinn besser begreifen zu können, verwendet BouRDIEU den Begriff der Strategie. Die objektiven und in ihrer inhärenten Logik verstandenen Strategien sind nicht bewusst und berechnend, weil sie eben verinnerlicht sind: sie verstehen sich wie von selbst.

«Der Habitus schließt die Lösung der Paradoxe des objektiven Sinns ohne subjektive Intention ein: er liegt jener Verkettung von 〈Zügen〉 zugrunde, die objektiv wie Strategien organisiert sind, ohne das Ergebnis einer echten strategischen Absicht zu sein - was zumindest voraussetzen würde, dass sie als eine von vielen möglichen Strategien aufgefasst werden» (Bourdieu 1987: 116).

Die Abwesenheit einer strategischen Absicht liegt im Habitus begründet, welcher den objektiven und vor- 
weggenommenen Strukturen angepasst ist, weil er von eben diesen gleichen Strukturen produziert wurde. Doch die objektiv strategischen Handlungen können auch versetzt sein, wenn die sozialen Strukturen sich verändern und nicht mehr denen gleichen, die verinnerlicht wurden (ibid).

Die Wirkung des Habitus auf menschliches Handeln hängt mit seiner Entstehung zusammen: Er ist das Ergebnis eines historischen Lernprozesses, die auf die Gegenwart wirkende Präsenz der gesamten Vergangenheit. Auf dieser Basis erzeugt er Strategien unter Rückbezug auf frühere Situationen und Geschichte, eine Art Repertoire an bewährten Praktiken. Damit macht der Habitus Praktiken relativ unabhängig von kurzfristigen äußeren Determiniertheiten der unmittelbaren Gegenwart. Er lässt sich von Routine und Routinisierung durch seinen nicht-subjektivistischen Einschlag abgrenzen: Routine setzt prinzipiell erst bei vom Subjekt/Individuum gemachten Erfahrungen ein, der Habitus ist aber ein gesellschaftlich vorgeprägtes Element, seine Routinisierung wirkt bereits bei den Wahrnehmungen und Entscheidungen als der Handlung vorausgehend.

Die Auffassung von der relativen Unabhängigkeit und Dauerhaftigkeit des Habitus und der ihm zugrunde liegenden Dispositionen ist speziell im Kontext von Entwicklungsgesellschaften interessant, die unter massiven externen Einflüssen stehen und einen besonders raschen sozialen Wandel durchlaufen. Zu fragen wäre hier, inwieweit interne Probleme dieser Gesellschaften auf Diskordanzen in der Veränderung äußerer Bedingungen und Habitus zurückgeführt werden können, gewissermaßen auf nicht mehr mit dem aktuellen Wandel konforme Dispositionen.

Das Konzept des Habitus erlaubt insgesamt ein besseres Verständnis der existierenden Unterschiede zwischen den in einem Feld interagierenden Handelnden, also auch zwischen jenen die man als Erben bezeichnen könnte und den Neuankömmlingen. Während die Einen den an das Feld angepassten Habitus besitzen, weil sie es seit ihrer frühesten Kindheit verinnerlicht haben, müssen sich die Anderen einen an das Feld angepassten Habitus neu definieren und aneignen (Mounier 2001: 73).

(5) Im Habitus liegen die Merkmale der Distinktion, welche sich in der räumlichen Organisation von Gesellschaften äußern.

Habitus ist zugleich Ergebnis sozialer Unterschiede wie auch Bedingung für deren Fortbestehen. Räumliche Bezüge werden dabei nicht unmittelbar erkennbar, zumal der Raumbegriff bei Bourdieu zumeist nicht im geographischen Sinne, sondern als Metapher für soziale Beziehungsgefüge («sozialer Raum») verwendet wird (Bourdieu 1991). Trotzdem kann man die gebaute Umwelt bzw. den «angeeigneten physischen Raum» durchaus als Ausdruck des Habitus derjenigen betrachten, die für die Gestaltung verantwortlich waren.

«Die im Habitus eingelagerten Klassifikationen und Unterscheidungsprinzipien, Bewertungs- und Denkschemata schlagen sich nieder in den Praxen der Lebensführung: vermittelt über den Habitus werden die Dinge - Wohnungen, Bücher, Autos, Kleidung, Kunstgegenstände, Besitztitel und so weiter - und die Aktivitäten - sportliche Betätigungen, kulturelle Aktivitäten, Reisen, Geselligkeiten - umgewandelt in $\langle[\ldots]$ distinkte und distinktive Zeichen〉, werden aus 〈kontinuierlichen Verteilungen [...] diskontinuierliche Gegensätze>, [...] geraten die Unterschiede aus der physischen Ordnung der Dinge in die symbolische Ordnung signifikanter Unterscheidungen» (BouRDIEU 1982: 284).

Auch wenn Bourdieu den physischen Raum, bzw. die räumliche Differenzierung nicht explizit in seine aus dem Habitus produzierten Distinktionen einbezogen hat, lässt sich argumentieren, dass Raumbezüge ebenfalls zur symbolischen Unterscheidung benutzt werden und sich Habitus insofern auch in der räumlichen Organisation der Gesellschaft äußert (HILLIER \& RoOKSBY 2002): Die Wahl des Wohnviertels in einer Stadt oder die Bevorzugung bestimmter Orte für das Einkaufen und Freizeitaktivitäten zählen zu den Unterscheidungsmerkmalen, welche über den Habitus vermittelt werden. Darüber hinaus wird die aktive Gestaltung von Räumen wesentlich durch den Habitus geprägt, der sich in den Feldern Architektur und Stadtplanung durchsetzen kann und der sich dadurch in baulicher Form dauerhaft materialisiert. Habitus tritt hier als Faktor auf, der das Wesen eines Ortes mitbestimmt. Schließlich wird auch das Verhältnis zwischen Menschen in den Ländern des Nordens und des Südens, die Art und Weise, wie sie voneinander denken und wie sie einander begegnen, durch Unterschiede im Habitus bestimmt.

Vor allem mit dem letzten Aspekt sind Themenbereiche von Relevanz für Entwicklungsländer angesprochen, die Fragen nach den forschungspraktischen Konsequenzen und der Operationalisierung der hier referierten konzeptionellen Überlegungen aufwerfen. Die wesentlichen Unterschiede zwischen subjektzentrierter und relationaler Handlungstheorie werden in Tabelle 2 noch einmal zusammengefasst.

\section{Konsequenzen für eine geographische Sozial- forschung in Entwicklungsländern}

Nach der zusammenfassenden Darstellung von Grundzügen der BourdiEuschen Sozialtheorie sei noch 


\begin{tabular}{|c|c|c|c|}
\hline & $\begin{array}{l}\text { Subjektzentrierte } \\
\text { Handlungstheorie } \\
\text { (GIDDENS 1997) }\end{array}$ & $\begin{array}{l}\text { Kritik an der } \\
\text { subjektzentrierten } \\
\text { Handlungstheorie }\end{array}$ & $\begin{array}{l}\text { Relationale und } \\
\text { kontextorientierte } \\
\text { Handlungstheorie } \\
\text { (BOURDIEU 1979) }\end{array}$ \\
\hline Perspektive & $\begin{array}{l}\text { Subjektzentriert, } \\
\text { methodologischer } \\
\text { Individualismus }\end{array}$ & $\begin{array}{l}\text { Gesellschaftliche } \\
\text { Bedingungen und } \\
\text { Beziehungen werden } \\
\text { weitestgehend } \\
\text { ausgeblendet. }\end{array}$ & $\begin{array}{l}\text { Subjekt von der } \\
\text { Strukturseite her ableiten } \\
\text { (z.B. Ökonomie, } \\
\text { Institutionen), von den } \\
\text { Bedingungen unter denen es } \\
\text { steht und dabei die Relation } \\
\text { in den Mittelpunkt stellen. }\end{array}$ \\
\hline \multirow[t]{3}{*}{ Prämissen } & $\begin{array}{l}\text { Akteure handeln «frei» } \\
\text { gemäß ihren Fähigkeiten. }\end{array}$ & $\begin{array}{l}\text { Akteure, vor allem jene in } \\
\text { Entwicklungsländern, } \\
\text { handeln im engen Rahmen } \\
\text { sozialer, ökonomischer und } \\
\text { ökologischer Bedingungen. }\end{array}$ & $\begin{array}{l}\text { Handlungen können nur } \\
\text { unter gesellschaftlichen } \\
\text { Bedingungen rekonstruiert } \\
\text { werden. }\end{array}$ \\
\hline & $\begin{array}{l}\text { Akteure handeln bewusst } \\
\text { und rational gemäß ihren } \\
\text { Ausstattungen. }\end{array}$ & $\begin{array}{l}\text { Akteure handeln u.a. } \\
\text { unbewusst (prädisponiert) - } \\
\text { aber immer «logisch». }\end{array}$ & $\begin{array}{l}\text { Rekonstruktion der } \\
\text { Eigenlogik des } \\
\text { Handlungssinns }\end{array}$ \\
\hline & Macht als Ressource & $\begin{array}{l}\text { Macht ist nicht extern, } \\
\text { sondern konstitutiv für jede } \\
\text { Form der } \\
\text { Vergesellschaftung. }\end{array}$ & $\begin{array}{l}\text { Machtverhältnisse und } \\
\text { soziale Positionen in die } \\
\text { Analyse integrieren, Subjekt } \\
\text { und Handlung daraus } \\
\text { ableiten. }\end{array}$ \\
\hline
\end{tabular}

Tab. 2: Die subjektzentrierte und die relationale Handlungstheorie im Vergleich

Comparison of the subject-related and relational theory of action

Comparaison de la théorie de l'action centrée sur le sujet et de la théorie relationnelle

einmal auf die im Eingangskapitel angeführten Kritikpunkte zu den Paradigmen Entwicklung und Handeln zurückgekommen. Vor diesem Hintergrund ergeben sich Fragen zum «Anregungspotenzial» BouRDIEUs für eine geographische Sozialforschung in Entwicklungsländern: Wie lassen sich die Konzepte Habitus und Feld in der empirischen Forschung operationalisieren? Welche neuen Fragestellungen ergeben sich daraus? In welcher Weise beziehen sie sich auf Sachverhalte, die bisher nicht oder anders untersucht wurden, und wie ist damit jeweils umzugehen? Wie müssten sich die Sicht- und Arbeitsweisen einer geographischen Sozialforschung in Entwicklungsländern von der bisherigen geographischen Entwicklungsforschung unterscheiden?
Um es gleich vorwegzunehmen: Erschöpfende Antworten auf diese Fragen können wir hier nicht geben, da wir die Auseinandersetzung mit den im ersten Kapitel angesprochenen Problemen als einen offenen Suchprozess verstehen, bei dem wir die Bourdieu-Rezeption lediglich als Orientierungshilfe sehen, aber nicht als Rezept für fertige Forschungsprogramme. Dennoch wollen wir zumindest in Ansätzen einige Antworten skizzieren.

Zunächst einmal sind zwei Hindernisse zu benennen, oder vielleicht besser: zwei Unbestimmtheiten, die dem Versuch, aus Bourdieus Überlegungen unmittelbar umsetzbare Anregungen für die empirische Forschung in Entwicklungsländern zu schöpfen, im Wege stehen. 
Die erste Unbestimmtheit besteht darin, dass BouRDIEU zwar seinen Ausgang von Untersuchungen in Nordafrika nahm, selbst aber nie seine Arbeiten als Beitrag zur Entwicklungstheorie verstanden hat. Seine konkreten Beispiele zu den Konzepten des Habitus und des Feldes stammen überwiegend aus dem Kontext der französischen Gesellschaft, auch wenn wichtige Vorarbeiten, beispielsweise zur Differenzierung der Kapitalsorten, bereits anhand von Untersuchungen in Algerien Anfang der sechziger Jahre angestellt wurden. Seine Theorie schließt also Gesellschaften in Entwicklungsländern durchaus ein, ist aber nicht exklusiv auf diese begrenzt. Die zweite Unbestimmtheit ist darin $\mathrm{zu}$ sehen, dass die zentralen Konzepte Habitus und Feld in Bourdieus Schriften abstrakt bleiben, zwar in verschiedenen Texten und Interviews variiert und umschrieben werden, aber doch eine präzise Ausführung als analytische Kategorien vermissen lassen. BourdiEu liefert keine griffigen Anweisungen zur empirischen Forschung, und auch seine «Theorie der Praxis» wird ausdrücklich nur als Entwurf bezeichnet. Der Schritt zur Umsetzung, die Konzepte gewissermaßen gebrauchsfertig für die Empirie $\mathrm{zu}$ machen, ist also noch zu leisten.

Aber vielleicht liegt gerade in den genannten Unbestimmtheiten eine Lösung für die beiden im ersten Kapitel erläuterten Kritikpunkte zur aktuellen Theoriediskussion. Zum ersten Kritikpunkt (Entwicklung): Bei Bourdieu geht es nicht um Entwicklung, sondern um Handeln im Kontext der Gesellschaft - und zwar in jeder möglichen Gesellschaft. Seine Konzepte sind grundsätzlich offen, also nicht auf bestimmte gesellschaftliche Formationen festgelegt. So vermeiden sie die Gefahr, den eurozentrischen Ballast des Entwicklungsdiskurses $\mathrm{zu}$ übernehmen, ohne selbst blind $\mathrm{zu}$ sein für die Probleme der Macht, der Ungleichheit oder der Unterdrückung, und bieten damit eine Alternative zu Theorien, die auf einem fragwürdigen Verständnis von Entwicklung aufbauen.

Zum zweiten Kritikpunkt (Handeln): Durch die Ablehnung des methodologischen Individualismus vermeidet Bourdieu die damit einhergehenden Probleme, weil sich der Fokus auf das Verhältnis von Individuum und Gesellschaft richtet, nicht auf das autonome Subjekt. Die Komplexität der sozialen Realität wird nicht reduziert, es wird nichts ausgeblendet, sondern der soziale Sinn, die Logik der Praxis im gesellschaftlichen Kontext werden in den Mittelpunkt des Interesses gerückt.

Daraus ergeben sich neue Fragestellungen, Forschungsperspektiven und Interpretationsansätze, die das Handeln der Subjekte einschließen, aber über individuelle Akteure hinausweisen und gesellschaftliche Prozesse in den Mittelpunkt stellen. Sie seien abschließend anhand einiger Beispiele skizziert.
In der Geschichte des europäischen Kolonialismus spielte der koloniale Habitus eine wichtige Rolle, ausgedrückt darin, dass die militärische und ökonomische Aneignung der Kolonien mit kultureller Überlegenheit, Sendungsbewusstsein und moralischer Verantwortung legitimiert wurde, dass das Verhältnis zwischen Kolonialherren und Kolonisierten von scharfen Distinktionen und Machtbeziehungen gekennzeichnet war, und dass die Transformation der außereuropäischen Gesellschaften so völlig selbstverständlich von europäischen Vorgaben bestimmt wurde (HINDESS 2002). Eine Analyse zur kolonialen Transformation Ägyptens zeigt (MrTCHELL 1988), in welcher Weise der koloniale Habitus als unhinterfragte gemeinsame Mission Tausender von Kolonialbeamten, Militärs und Ingenieuren innerhalb von wenigen Jahrzehnten das Land zu einer Art riesigen Maschinerie umgestaltete. Ein weiteres Beispiel für die Auswirkungen eines kolonialen Habitus findet sich in einer Studie über die Unterdrückung der Aborigines in Australien, denen noch in der ersten Hälfte des 20. Jahrhunderts auf staatliche Veranlassung mehr als 100'000 Kinder gewaltsam weggenommen wurden, um sie in westlichem Sinne zu «zivilisierten» Menschen zu machen (GALE 2002).

Auch in der Gegenwart ist das Verhältnis zwischen Menschen aus Nord und Süd durch ein Gefälle geprägt, das sich nicht allein auf ökonomische Disparitäten beschränkt, sondern in der Nachfolge des kolonialen Habitus steht. So könnte man heute den gesamten Bereich der internationalen Entwicklungszusammenarbeit im BouRdiEuschen Sinne als ein mehrfach gestaffeltes soziales Feld betrachten, in dem eine Vielzahl von Akteuren um Macht, Einfluss und Ressourcen konkurrieren. Vordergründig geht es ihnen allen um Entwicklung, aber de facto dient ihr Handeln auch ihrer Positionierung im Feld, d.h. der Sicherung und Ausdehnung von Einflusssphären und der Durchsetzung bestimmter Vorstellungen von Entwicklung. Die Frage ist, in welcher Weise sich ein spezifischer Habitus in Bezug auf dieses Feld ausprägt, inwiefern er im Handeln der Beteiligten zum Ausdruck kommt und womöglich auch heute noch den Verlauf des Spiels um Entwicklung beeinflusst.

Zum Schluss sei noch ein Beispiel aus einem laufenden Forschungsprojekt angeführt, das zeigen soll, wie die Konzepte des Habitus und des symbolischen Kapitals auch auf der Ebene lokaler Fallstudien zur Interpretation beobachteter Praktiken herangezogen werden können: Arbeitsmigranten aus dem Hohen Atlas in Südmarokko investieren massiv in den Bau neuer Häuser, Pisten, Moscheen und Wasserspeicher in ihren Herkunftsdörfern. Der Umfang der Investitionen ist ökonomisch schwer erklärbar, da ein großer Teil des neu geschaffenen Wohnraumes leer steht, nur ein Teil der Migranten tatsächlich wieder in die 
Heimatorte zurückkehrt, und die Dörfer auch schon zuvor mit Moscheen versorgt waren. Die intensive Bautätigkeit lässt sich unschwer als ein auf Prestigegewinn ausgerichteter Schauwettkampf interpretieren, aber auffällig ist dabei, dass die Migranten-Familien sehr unterschiedlich an dieser symbolischen Konkurrenz beteiligt sind, und zwar weitgehend unabhängig von ihrer Finanzkraft. Die Rationalität ihres Investitionsverhaltens lässt sich weder ökonomisch erklären, noch würde hier eine ausschließlich subjektzentrierte Handlungsanalyse weiterhelfen, sondern entscheidend ist die Berücksichtigung der Beziehungen zwischen den Akteuren und ihrer gesellschaftlichen Umgebung. Die objektiven Relationen, sozialen Positionen und Machtbeziehungen erklären die Handlungsweisen der sog. Gastarbeiter und der im Dorf Verbliebenen, ohne dass diese sich dessen immer bewusst sein müssen.

Protagonisten der Bautätigkeit sind nämlich vor allem die Angehörigen der untersten sozialen Schichten, die Nachkommen ehemaliger Leibeigener und Landarbeiter, die ihr während der Arbeitsmigration akkumuliertes «ökonomisches Kapital» in Form von prunkvollen Häusern zu «symbolischem Kapital» umwandeln, um damit in der stark hierarchisierten ländlichen Gesellschaft gegenüber den Gruppen mit ererbtem symbolischen Kapital (z.B. Nachkommen des Propheten) aufzusteigen. Diese Strategie der Transformation der Kapitalsorten ist für die Migranten deshalb so wichtig, weil andere Wege der Kapitalumwandlung begrenzt sind, aber sie ist auch konfliktträchtig, weil die alten Eliten versuchen, den status quo der sozialen Ordnung gegen das Streben der Migranten nach Anerkennung $\mathrm{zu}$ verteidigen.

Aus der hier skizzierten Interpretation ergeben sich eine Vielzahl von weiterführenden Fragen: Welche anderen nicht-ökonomischen Mittel nutzen die Nachkommen der ehemaligen Leibeigenen und Landarbeiter, um symbolisches Kapital in ihren Herkunftsdörfern $\mathrm{zu}$ erwerben? Welche matrimonialen und politischen Strategien verfolgen sie, um ihre soziale Position aufzuwerten? Hier könnte sich der Habitus der dominierten und bisher vom Zugang zu symbolischem Kapital ausgegrenzten Sozialgruppe, d.h. ihre inkorporierte Geschichte und verinnerlichten Dispositionen, aufschlussreich zeigen, um zu verstehen, warum sie trotz einer zum Teil deutlich verbesserten ökonomischen Situation nicht ohne weiteres ihre subordinierte soziale Position überwinden können.

Fazit: BouRDIEUs Werk bietet theoretische Anregungen für den Versuch, das Handeln nicht nur vom Subjekt aus zu betrachten, sondern stärker die gesellschaftliche Ebene und speziell das Verhältnis zwischen der Gesellschaft und den Akteuren (agents) in den Mittelpunkt zu stellen. Abhängigkeiten und Machtverhältnisse sind keine Randbedingungen, sondern sie sind zentral, um die gesellschaftliche Praxis verstehen zu können. Da sie hier nicht als substantielle Kategorien gedacht werden (Ressourcen), muss vielmehr der Gehalt dieser Machtbeziehungen auf einer symbolisch-performativen Ebene rekonstruiert werden. Diese Ausweitung der Perspektive könnte einen Ausweg aus der Einseitigkeit des Entwicklungsdiskurses und aus der Begrenztheit des methodologischen Individualismus weisen. Dazu bedürfen die Konzepte Habitus und Feld jedoch der Operationalisierung im Rahmen einer geographischen Sozialforschung in Entwicklungsländern.

\section{Literatur}

Asendorf, M., Flemming, J., von MÜller, A. \& V. UlLRICH (Hrsg.) (1994): Geschichte. Lexikon der wissenschaftlichen Grundbegriffe. - Reinbek: Rowohlt.

Blotevogel, H.H. (1999): Sozialgeographischer Paradimenwechsel? Eine Kritik des Projekts der handlungszentrierten Sozialgeographie von Benno Werlen. - In: Meusburger, P. (Hrsg.): Handlungsorientierte Sozialgeographie: Benno Werlens Entwurf in kritischer Diskussion. $-=$ Erdkundliches Wissen 130, Stuttgart: 1-33. BoeckH, A. (1992): Entwicklungstheorien: Eine Rückschau. - In: Nohlen, D. \& F. Nuscheler (Hrsg.): Handbuch der Dritten Welt. - Bonn, J.H.W. Dietz Verlag: 110-130.

BoHLE, H.-G. (2001): Neue Ansätze der geographischen Risikoforschung. Ein Analyserahmen zur Bestimmung nachhaltiger Lebenssicherung von Armutsgruppen. In: Die Erde 132: 119-140.

Boнn, C. (1991): Habitus und Kontext. - Opladen: Leske \& Budrich.

Bourdieu, P. (1979): Entwurf einer Theorie der Praxis. - Frankfurt am Main: Suhrkamp.

BouRdieu, P. (1982): Die feinen Unterschiede. Kritik der gesellschaftlichen Urteilskraft. - Frankfurt am Main: Suhrkamp.

Bourdieu, P. (1987): Sozialer Sinn. Kritik der theoretischen Vernunft. - Frankfurt am Main: Suhrkamp.

BourdiEu, P. (1991): Physischer, sozialer und angeeigneter physischer Raum. - = In: WentZ, M. (Hrsg.): StadtRäume. - Frankfurt, New York: Campus Verlag: 25-34. Bourdieu, P. (1992): Rede und Antwort. - Frankfurt am Main: Suhrkamp.

Bourdieu, P. (2000): Die zwei Gesichter der Arbeit. Interdependenzen von Zeit- und Wirtschaftsstrukturen am Beispiel einer Ethnologie der algerischen Übergangsgesellschaft. - Konstanz: UVK-Verlagsgesellschaft (franz. Original: Algérie 60. Structures économiques et structures temporelles. - Paris: Edition de Minuit 1977).

Bourdieu, P., Chamboredon, J.-C. \& J.-C. Passeron (1973): Grundlagen einer Theorie der symbolischen Gewalt. - Frankfurt am Main: Suhrkamp.

Bourdieu, P., Chamboredon, J.-C. \& J.-C. Passeron 
(1991): Soziologie als Beruf. Wissenschaftstheoretische Voraussetzungen soziologischer Erkenntnis. - Berlin, New York: de Gruyter.

Bourdieu, P. \& L.J.D. WacQuant (1996): Reflexive Anthropologie. - Frankfurt am Main: Suhrkamp.

Elsenhans, H. (1996): Kein Ende der großen Theorie. - In: Asien-Afrika-Lateinamerika 2: 111-146.

Elsenhans, H. (1997): Gegen den Theoriepessimismus in der Entwicklungspolitik. - In: ZAPOTOCZKY, K. \& P.C. Gruber (Hrsg.): Entwicklungstheorien im Widerspruch. - Frankfurt am Main: Brandes \& Apsel: 73-97. Foucault, M. (2002): Der Wille zum Wissen. Sexualität und Wahrheit, Bd. I. - Frankfurt am Main: Suhrkamp. Gale, F. (2002): The Endurance of Aboriginal Women in Australia. - In: Hillier, J. \& E. Rooksby (Hrsg.): Habitus: a sense of place. - Burlington: Ashgate Verlag: 339-351.

Giddens, A. (1997): Die Konstitution der Gesellschaft. Grundzüge einer Theorie der Strukturierung. - 3. Auflage, Frankfurt am Main, New York: Campus Verlag.

HeIn, W. (1997): Das «Scheitern der Großen Theorie», der Globalisierungsproze $\beta$ und die Krise nationaler Gesellschaften - Ein Thesenpapier. - In: Peripherie 65/66: 109-132.

Hillier, J. \& E. Rooksby (Hrsg.) (2002): Habitus: a sense of place. - Burlington: Ashgate.

Hindess, B. (2002): Metropolitan Liberalism and Colonial Autocracy. - In: Hillier, J. \& E. Rooksby (Hrsg.): Habitus: a sense of place. - Burlington: Ashgate: 101-114. ILLICH, I. (1970): Die Kehrseite der Barmherzigkeit. = In: Illich, I. (Hrsg.): Almosen und Folter. Verfehlter Fortschritt in Lateinamerika. - München: Kösel Verlag: 43-55.

KöSSLER, R. (1998): Entwicklung. - = Einstiege 3, Grundbegriffe der Sozialphilosophie und Gesellschaftstheorie. - Münster: Westfälisches Dampfboot.

Krais, B. \& G. Gebauer (2002): Habitus. - Bielefeld: transcript Verlag.

Kreutzmann, H. (2002): Zehn Jahre nach Rio. (Wieder-) Entdeckung der Armut oder Entwicklungsfortschritte im Zeitalter der Globalisierung. - In: Geographische Rundschau 54, 10: 58-63.

KRINGS, T. (1991): Agrarwissen bäuerlicher Gruppen in Mali/Westafrika. - = Abhandlungen - Anthropogeographie, Sonderheft 3, Berlin: 1-301.

KRINGS, T. \& B. MüLler (2001): Politische Ökologie: theoretische Leitlinien und aktuelle Forschungsfelder. - In: Reuber, P. \& G. Wolkersdorfer: Politische Geographie. Handlungsorientierte Ansätze und Critical Geopolitics. - = Heidelberger Geographische Arbeiten 112, Heidelberg: 93-116.

Mbembe, A. (2001): On the postcolony. - Berkeley, Los Angeles, London: University of California Press.

MenZel, U.(1992): Das Ende der Dritten Welt und das Scheitern der großen Theorie. - Frankfurt am Main: Suhrkamp. Meusburger, P. (1999): Subjekt - Organisation Region. Fragen an die subjektzentrierte Handlungs- theorie. - In: Meusburger, P. (Hrsg.): Handlungsorientierte Sozialgeographie: Benno Werlens Entwurf in kritischer Diskussion. - = Erdkundliches Wissen 130, Stuttgart: 95-132.

Mitchell, T. (1988): Colonising Egypt. - Cambridge: Cambridge University Press.

Mounier, P. (2001): Pierre Bourdieu, une introduction. - Paris: La Découverte.

MÜller-Mahn, D. (2001): Fellachendörfer. Sozialgeographischer Wandel im ländlichen Ägyten. - = In: Erdkundliches Wissen 127, Stuttgart: Steiner.

Neuburger, M. (2002): Pionierfrontentwicklung im Hinterland von Cáceres (Mato Grosso, Brasilien). Ökologische Degradierung, Verwundbarkeit und kleinbäuerliche Überlebensstrategien. - = Tübinger Geographische Studien 135, Tübingen.

Nohlen, D. \& F. Nuscheler (1992): Ende der Dritten Welt? - In: Nohlen, D. \& F. Nuscheler (Hrsg.): Handbuch der Dritten Welt. - Bonn: J.H.W. Dietz Verlag: 14-30.

SACHS, W. (1989): Zur Archäologie der Entwicklungsidee. - In: epd-Entwicklungspolitik 10: a-i.

Scholz, F. (1995): Nomadismus. Theorie und Wandel einer sozio-ökologischen Kulturweise. - = Erdkundliches Wissen 118, Stuttgart: Steiner.

Scholz, F. (2002): Die Theorie der «fragmentierenden Entwicklung». - In: Geographische Rundschau 54, 10: 6-11.

SEers, D. (1974): Was heißt «Entwicklung»? - In: SENGHAAS, D. (Hrsg.): Peripherer Kapitalismus. Analysen über Abhängigkeit und Unterentwicklung. - Frankfurt am Main: Suhrkamp Verlag: 39-67.

TRAORÉ, A.D. (2002): Afrikanische Nachhaltigkeit. Was die Entwicklungspolitik nicht wissen will. - In: Le Monde Diplomatique 9: 15.

TRÖGER, S. (2002): Gesellschaftliche Umverteilung, ein moralisches Muss? Verwundbarkeit und soziale Sicherung in Zeiten gesellschaftlichen Umbruchs - Beobachtungen aus Tansania in akteursorientierter Interpretation. - In: Geographica Helvetica 1:34-45.

WERLEN, B. (1995): Sozialgeographie alltäglicher Regionalisierungen. - = In: Erdkundliches Wissen 116 \& 119, Stuttgart: Steiner.

WerLen, B. (1999): Handlungszentrierte Sozialgeographie. Replik auf die Kritiken. - = In: Meusburger, P. (Hrsg.): Handlungsorientierte Sozialgeographie: Benno Werlens Entwurf in kritischer Diskussion. - = Erdkundliches Wissen 130, Stuttgart: 247-268.

Werlen, B. (2000): Sozialgeographie. - Bern u.a.: Paul Haupt-Verlag.

WiRTH, E. (1998): Handlungsgeographie als Königsweg einer modernen Regionalen Geographie. - In: Geographische Rundschau 51, 1: 57-64.

ZIAI, A. (2001): Post-Development: Perspektiven für eine afrikanische Debatte? - = In: Focus Afrika, IAKDiskussionsbeiträge 18, Institut für Afrika-Kunde, Hamburg: 1-49. 


\section{Zusammenfassung: Habitus und Feld - Anregungen für eine Neuorientierung der geographischen Entwicklungsforschung auf der Grundlage von Bourdieus «Theorie der Praxis»}

Der Beitrag plädiert für eine Neuorientierung der geographischen Entwicklungsforschung im Sinne einer geographischen Sozialforschung in Entwicklungsländern. Ausgangsbasis dafür ist eine kritische Auseinandersetzung mit der aktuellen entwicklungstheoretischen Diskussion und der Übertragung akteurszentrierter Ansätze in die Entwicklungsforschung. Die Kritik an diesen Ansätzen konzentriert sich auf deren unzureichend fundiertes Gesellschaftsverständnis, in dem die Beziehung zwischen Subjekt und Gesellschaft nicht hinreichend erklärt wird und Abhängigkeitsverhältnisse $\mathrm{zu}$ wenig berücksichtigt werden. Mit der «Theorie der Praxis» von Pierre BourDIEU wird versucht, die Forschungsperspektive auf genau jene gesellschaftsrelevanten Zusammenhänge zu richten. Im Mittelpunkt stehen dabei das «soziale Feld», das durch Machtbeziehungen und die Konkurrenz von Akteuren geprägt wird, und der «Habitus» als System dauerhafter Dispositionen bzw. als verinnerlichte Verhaltensgrammatik. Nach der Darstellung der wichtigsten Bourdieuschen Theoreme schließt der Artikel mit einigen kurz umrissenen Beispielen, die eine mögliche forschungspraktische Umsetzung skizzieren.

\section{Résumé: Réflexions en vue d'une nouvelle orienta- tion de la recherche géographique en matière de développement, à partir de la «Théorie de la prati- que» de Bourdieu}

Cette contribution plaide en faveur d'une réorientation de la géographie du développement vers une recherche géographique sociale dans les pays en développement. Elle est fondée d'une part sur un examen critique du débat actuel sur les théories du développement, d'autre part sur celui du transfert, dans la recherche sur le développement, des concepts centrés sur les acteurs. La critique adressée à ces concepts se focalise sur une conception trop réduite de la société, qui ne permet pas une analyse suffisamment approfondie du rapport entre le sujet et la société et qui ne prend pas assez en considération les relations de dépendance. La contribution entend orienter précisément les perspectives de recherche vers ces rapports si importants pour la compréhension d'une société, à l'aide de la «théorie de la pratique» de Pierre Bourdieu. Le «champ social», façonné par les rapports de pouvoirs et une compétition entre agents, et «l'habitus», en tant que structure de dispositions durables et grammaire comportementale intériorisée, sont ainsi placés au centre de cette démarche. Après une présentation des principaux théorèmes de Bourdieu, l'article esquisse, à partir de quelques exemples, une mise en œuvre possible de cette approche dans le domaine de la recherche.
Summary: Habitus and Field - Impulses for a reorientation of geographical development theory based on Bourdieu's «Theory of Practice»

The article recommends reorienting geographical development research toward geographical social research in developing countries. The recommendation is based on a critical review of the current debate over development theories and, more specifically, the application of actor-oriented approaches in development research. The critique of these approaches focuses on their narrow concept of society, which does not allow for a profound analysis of the relationship between subject and society, and which does not adequately consider socio-economic dependencies. PIERRE BOURDIEU's «theory of practice» allows a redirection of research towards societal relationships. The key concepts of Bourdieu's theory are «social field», which is formed by relations of power and the socio-political competition between actors, and «habitus», which is conceived as a system of lasting dispositions and an internalized behavioral «grammar». After the presentation of Bourdieu's most important theorems, the article closes with brief examples to indicate their possible implementations in research.

\section{Didaktische Hinweise}

- Worauf bezieht sich die Kritik des Artikels an der aktuellen entwicklungstheoretischen Diskussion und der geographischen Entwicklungsforschung?

- Welche Argumente werden aufgeführt, um eine stärker gesellschaftsbezogene «geographische Sozialforschung in Entwicklungsländern» zu begründen?

- Was beinhaltet der livelihood-Ansatz und welches sind Kritikpunkte an demselben?

- Was versteht man unter dem Bourdieuschen Begriff «Habitus»?

- Worin besteht der Zusammenhang zwischen den zentralen Begriffen der «Logik der Praxis»?

- Welche Beispiele lassen sich für den «Habitus» anführen?

M.A.Thomas Dörfler,Dr. Olivier Graefe,Prof.Dr.Detlef Müller-Mahn, Lehrstuhl für Bevölkerungs- und Sozialgeographie der Universität Bayreuth, Universitätsstrasse 30, D-95447 Bayreuth.

e-mail:

Thomas.Doerfler@uni-bayreuth.de

Olivier.Graefe@uni-bayreuth.de

muellermahn@uni-bayreuth.de

\section{Manuskripteingang/received/manuscrit entré le} 21.11.2002

Annahme zum Druck/accepted for publication/accepté pour l'impression: 3.4.2003 